\title{
APORTE CRÍTICO AL ANÁLISIS DE LA NORMATIVIDAD AMBIENTAL
}

María Helena Huertas estudiante de Ingeniería Ambiental Universidad de los Andes.

David Vásquez Cardona Ingeniero Agrónomo Universidad de Caldas

Integrantes del Centro de Investigación de pensamiento latinoamericano Raíz-al. dvsaudadeazul@gmail.com

todo progreso realizado en la agricultura capitalista, no es solamente progreso en el arte de esquilmara al obrero, sino también en el arte de esquilmar a la tierra ...la producción capitalista sólo sabe desarrollar la técnica socavando al mismo tiempo las dos fuentes originaria de toda riqueza: la tierra y el hombre Carlos Marx. El Capital

Manizales, 2008-10-30 (Rev. 2008-11-28)

\section{RESUMEN}

La normatividad ambiental internacional, que surge desde la declaración de Estocolmo en 1972, no ha logrado disminuir la contaminación ambiental sino que, como se observa en los más diversos informes especializados sobre el tema, los peligros que enfrenta la vida en el planeta son cada vez más inminentes. Lo anterior se debe a una comprensión errada del problema, pues no consiste -como es presentado en Nuestro Futuro Común- en la tecnología en sí misma, sino en el desarrollo del modo de producción que corresponde al modo de vida desarrollado en la sociedad burguesa; tema que no es analizado de manera crítica en ninguno de los discursos elaborados desde los organismos multilaterales. De igual forma, gran parte de las normas técnicas construidas como el tratado de Kyoto, a pesar de ser limitadas en sus alcances, no han logrado ser implementadas debido a que el poder económico de las naciones y de los grandes consorcios define por prioridad sus ganancias por encima de la vida.

\section{PALABRAS CLAVES}

Normatividad, tecnología, producción, sociedad burguesa.

\section{CRITICAL CONTRIBUTION TO THE ANALYSIS OF ENVIRONMENTAL NORMATIVITY}

\begin{abstract}
International environmental regulations that emerged from the Stockholm Declaration adopted in 1972 haven't been able to reduce environmental pollution; rather, as observed in the most diverse specialized reports, the dangers to life on the
\end{abstract}


planet are increasing. This is because environmental problems have been misunderstood, since the problem doesn't consist in, as presented in Our Common Future, in technology itself, but in the development of the mode of production in the bourgeois society. However, this element is not critically analyzed in any of the speeches from multilateral agencies. Similarly, several technical regulations such as the Kyoto Protocol, in spite of being limited, have not been implemented because of the economic power of nations and because big companies prioritize their earnings over life.

\section{KEY WORDS}

Regulations, technology, production, bourgeois society.

\section{INTRODUCCIÓN}

La crisis de la vida es el diagnóstico elaborado por los más diversos informes técnicos ${ }^{1}$, más no existe de parte de las organizaciones multilaterales y estatales que elaboran muchos de los informes- una crítica radical que se sumerja en las raíces del problema. El propósito es exponer los motivos por los cuales las políticas ambientales trazadas por la Organización de las Naciones Unidas, a 35 años de la declaración de Estocolmo, no han cambiado en nada la crisis ambiental del planeta, por el contrario, los encuentros que se han venido realizando y las declaraciones de los mismos que van hasta Johannesburgo en el 2006, no han hecho más que reafirmar la agudización progresiva de la crisis hasta el punto de ver amenazada la vida en la tierra tal cual la conocemos. Nos preguntamos ¿la agudización de la crisis se debe a un error en el abordaje del análisis de las diversas declaraciones o se debe a un vacío de poder para la ejecución de las determinaciones? Para resolver la pregunta es necesario detenerse en las diversas declaraciones y encontrar el abordaje metódico del problema, realizando un acercamiento a las determinaciones trazadas.

\section{EL CLUB DE ROMA}

Un primer análisis que advirtió a la comunidad internacional sobre la crisis ambiental fue el presentado por el Club de Roma -asociación privada compuesta por hombres de empresa, científicos y participantes en la vida pública internacional- titulado Los Límites del Crecimiento el cual estuvo a cargo del instituto de Tecnológico de Massachusetts y bajo la dirección de Dennis L. Meadows, en el cual, además de presentar un panorama bastante gris, creen realizar un gran aporte metodológico por encontrar una manera de contrastar y medir las diversas variables que para ellos se interrelacionan en la problemática ambiental. La propuesta metodológica consistía en el desarrollo de la modelación de sistemas ${ }^{2}$, bajo la cual unas variables determinadas se pueden proyectar en el tiempo realizando unas hipótesis bajo las que se predice el comportamiento de cada variable. Sobre esta herramienta, de manera inocente, se pretende alcanzar un análisis objetivo de la realidad y, por lo tanto, desarrollar una serie de consideraciones de acuerdo a la predicción lograda por el programa de modelación. Meadows y sus acompañantes desconocen por completo que la formulación de las hipótesis bajo las cuales se proyectan las variables obedecen a su criterio y que, por lo tanto, la objetividad no está dada por la herramienta de medición, sino por su capacidad para acercarse a la realidad del objeto, pues el sometimiento de la realidad del objeto a la experimentación o a una herramienta de medición está dada por la modelación que realiza el sujeto. La postulación que la contrastación de los objetos a un modelo de experimentación 
determina la objetividad y la verdad, obedece al materialismo mecánico y al positivismo que privilegian la descripción, la cuantificación y la predicción a la explicación, a la ordenación de la realidad por medio del concepto y la teoría.

Al volver sobre el positivismo Meadows y su equipo, se olvidan de analizar críticamente las premisas sobre las cuales elaboran las hipótesis, es así que afirman que el problema ambiental está relacionado directamente con la sobrepoblación ${ }^{3}$; para ellos hay una relación directa entre el crecimiento económico y el crecimiento poblacional, que son los variables sobre las que trazan la interacción de la contaminación, la producción de alimentos y el agotamiento de recursos naturales. En su análisis "la población no puede crecer sin alimentos, la producción de alimentos aumenta con el crecimiento de capital, el capital exige más recursos, los recursos desechados se convierten en contaminación, la contaminación interfiere en el crecimiento de la población y de los alimentos"4. Lo anterior deja ver como su lógica formal busca una secuencia de causas que de cuenta de de los factores producto de la contaminación y sus repercusiones en la demás variables, más pese a sus esfuerzos no logran desencadenar el problema y presentarlo como un todo, por lo que terminan presentando como centro del problema el crecimiento poblacional. El desconocimiento de la historia y del desencadenamiento de los hechos a la hora de abordar el estudio es una de las grandes falencias cometidas por los integrantes del Instituto Tecnológico de Massachussets, si bien reconocen que el inicio del problema se presenta con la industrialización, más no ponen en el centro de la discusión los modos de producción, que contienen la forma en la que el hombre se relaciona con la naturaleza y consigo mismo como especie. La relación hombre naturaleza que se ve contenida en el modo de producción capitalista está determinada por una actuar violento, que busca esquilmar paso a paso la vida natural en beneficio de los intereses privados bajo los cuales opera la sociedad burguesa, cuestión desentendida por el Club de Roma.

Los modos de producción capitalista que corresponden a la sociedad burguesa sustentan su ejercicio en romper los ciclos energéticos de los ecosistemas al construir paisajes modificados que extraen parte de la energía útil disponible de la naturaleza que luego se ve convertida energía que es entendida inmediatamente como un desecho, pues las mercancías cuando pierden su valor de uso son residuos indeseables debido a un pensamiento precario que no ve la necesidad de retribuirle a la tierra lo que le ha sido quitado, acelerando así el proceso entrópico natural de la energía, que la vida en los ecosistemas naturales busca bajo todas las formas aprovechar al máximo, evitando la disipación de la energía a través de la cadena trófica y conservándola por medio de la genética ${ }^{5}$. El quitarle y no restablecer lo tomado a la naturaleza, y el desarrollo de la extracción de materiales fósiles como combustibles, sin pensar en sus derivados, es el principal problema ambiental, lo que es desestimado en "los límites del crecimiento" debido a que dicho análisis carece de una comprensión teórica, propia de la lógica y el método que devele el modo de producción, por lo cual se queda en una simple descripción de hechos y circunstancias propias del aparecer de los fenómenos.

Si bien la población es un tema fundamental en el análisis ambiental, ésta no puede ser vista como una simple variable cuantitativa, puesto que las comunidades, las poblaciones humanas, son productoras de cultura y de vida, se apropian de los medios que los rodean de acuerdo a una conciencia que han elaborado del mundo a través del conocimiento (ciencia en su forma más elaborada) y la técnica, siendo esa apropiación que realizan del mundo la que determina los modos de producción, permitiendo a su vez la reproducción material como especie y define el relacionamiento con la vida y la naturaleza ${ }^{6}$. 
Meses después de la publicación "Ios límites del crecimiento", en junio del mismo año la Organización de Naciones Unidas celebra en Estocolmo su primera reunión para analizar la crisis ambiental que vive el planeta, en la cual elabora una declaración que busca ser una inspiración para "preservar y mejorar el medio humano". Lastimosamente el análisis se centra en el medio humano y no en la vida del planeta: "Los dos aspectos del medio humano, el natural y el artificial, son esenciales para el bienestar del hombre y para el goce de los derechos humanos fundamentales, incluso el derecho a la vida misma"7. En esta perspectiva la tierra y los seres que en ella existen, tanto inertes como vivos, están al servicio del hombre, lo que deja ver la continuidad del pensamiento planteado por Bacon en el siglo XVII donde los hombres son amos de la naturaleza, dejando de ser su cuerpo inorgánico del que dependen para no morir, para convertirse en su propiedad, en su medio. Al respecto nos señala Bacon: "pero si nos dejásemos guiar por ella en la invención, podríamos ser sus amos en la práctica"8. Lo anterior no es más que producto del divorcio hombre y naturaleza, obligado por el afán de poder que se encuentra en la base del modelo civilizatorio de occidente donde se privilegia por encima del conocimiento y de la verdad, el facto, las herramientas para controlar el mundo natural asegurando el beneficio egoísta que se encuentra en el seno de los valores de la sociedad burguesa. Así, el intelecto no busca conocer la naturaleza sino desarrollar las herramientas para dominarla, lo que hace inviable el camino donde la acción guiada por el pensar encuentre la pauta para la existencia común de la especie humana y de la vida natural.

\section{LOS PRINCIPIOS DE LA NORMATIVIDAD}

Se anuncia en Estocolmo que el daño del "medio humano" es infligido por el mismo hombre dado sus avances en las ciencias y la tecnología, sin por ello dejar de reconocer que dichos avances pueden llevar al hombre a superar las necesidades materiales como especie, textualmente señala: "Hoy en día, la capacidad del hombre de transformar lo que lo rodea, utilizada con discernimiento, puede llevar a todos los pueblos los beneficios del desarrollo y ofrecerles la oportunidad de ennoblecer sus existencia. Aplicado errónea o imprudentemente, el mismo poder

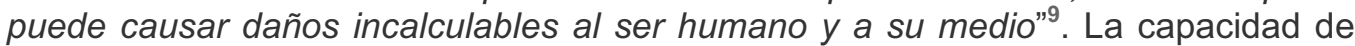
discernimiento, el poder para transformar la naturaleza no es, según lo expuesto el problema, sino el uso que se le da a dicha capacidad, lo que implica que son los intereses, la voluntad de los hombres, lo que nos puede conducir al deterioro de la vida o a su preservación, el juicio expuesto indica por dónde abordar el problema, pero no se continua en el documento dicho análisis. Sin embargo, el párrafo citado obvia que la capacidad de transformación, además de estar determinada por unos intereses, está definida por el conocimiento que se tiene sobre el objeto, y si éste es de carácter parcial como lo implica el saber empirista que guía el mito de la ilustración -donde lo importante no es el saber sino la conquista y la transformación para un beneficio particular- las herramientas aplicadas para lograr el fin obvian las consecuencias. Así, los intereses y el conocimiento que privilegia el facto sobre la verdad son la esencia del problema que escapa al análisis de Estocolmo, es por eso que las propuestas esbozadas en los principios son limitadas y evaden la discusión económica y política inmersas en el problema, evadiendo las relaciones de poder entre las naciones y los sectores industriales y financieros, planteando que el equilibrio natural puede convivir con la dinámica de acumulación de capital propia de los modos de producción de la sociedad burguesa.

Luego de Estocolmo, las Naciones Unidas dan paso a la conformación de un grupo de expertos en el tema conocido como la comisión Brundtland, el cual, reunido desde el año 1983, logra elaborar en el transcurso de su ejercicio de análisis el documento Nuestro Futuro Común, publicado en el año de 1987, donde se expone 
el concepto desarrollo sostenible que ha sido pionero en la construcción de las bases ambientales a nivel legislativo. El desarrollo sostenible afirma que se debe "asegurar que la humanidad satisfaga las necesidades del presente sin comprometer la capacidad de las futuras generaciones para satisfacer las propias"10. Propone que para lograr una sintonía entre el modelo de desarrollo capitalista y el ambiente, es necesario que se conquisten soluciones tecnológicas ambientalmente viables para estimular la producción (lo que evidencia la contradicción de su discurso, pues en dicho documento se expone en la presentación del problema que el desarrollo conocido de las fuerzas productivas es un modelo inviable ambientalmente), dejando así en manos de la ciencia y la tecnología la solución del problema ambiental, poniendo a la ciencia y a la tecnología como las portadoras de soluciones es sí mismas, cuando, como ya se ha señalado, las construcciones que se dicen ser científicas en el mito iluminista tienen de antemano la plena intención de controlar, más no de conocer, lo que no garantiza la salida a la problemática generada, pues los logros no están basados en la conciencia de la realidad, sino en los desarrollos de la industria que son los que promueven las conquistas "científicas" y técnicas funcionales al modo de producción. Así, a la ciencia anclada en el pensamiento positivista le es imposible conquistar la reconciliación soñada en nuestro futuro común, en donde la "ciencia se percibe como neutral, tanto desde el punto de vista político como desde el ético"11.

El concepto de desarrollo sostenible mantiene la visión del hombre como sujeto que manipula la naturaleza a su favor para preservar la especie y generar mayor desarrollo, dejando pre-establecido que el estilo de vida al cual se encuentra sujeto el hombre actualmente es sostenible de por si $^{12}$.

Contiene el informe una noción problemática de América latina, al mencionar que su desarrollo en el modelo de crecimiento actual genera mayor degradación de los ecosistemas, destacando que los inadecuados usos de los recursos por partes de las poblaciones pobres -debido a su escasez de conocimientos- provocan un impacto negativo sobre el ambiente, con lo cual se oculta que los principales responsables del deterioro ambiental son los países del "Primer Mundo" quienes poseen la mayor cantidad de industrias y promueven la extracción de materias primas del Tercer Mundo a cualquier costo, dejando como consecuencia una deuda biológica, en la mayoría de los casos, irrecuperable.

Las declaraciones de Río en el año 1992, titulada como La Carta a la Tierra y la declaración de Johannesburgo del 2006, se centran sobre el mismo principio de desarrollo sostenible postulado en nuestro Futuro Común, razón por la cual, más allá de pequeños avances en la construcción de acuerdos y políticas públicas a través de la Agenda 21, definida en Río,y expresiones de la forma en la que se agudiza la manifestación de la crisis ambiental, no existe un cambio en el análisis ni en la construcción de soluciones, por lo que las propuestas son limitadas y sin la efectividad para transformar el panorama.

\section{NORMAS TÉCNICAS}

Los protocolos técnicos, que surgen como propuestas prácticas para superar los efectos de la crisis, sólo han logrado tener efectividad cuando comprometen a todos los países de las Naciones Unidas, el caso más representativo es el Protocolo de Montreal de 1987, que vinculó a todos los países del mundo y el cual prohibió el uso de determinados gases (ej. CFC's) causantes de la destrucción de la capa de ozono, independientemente de las implicaciones económicas que representara para las naciones el dejar de utilizar dichas sustancias. En cambio protocolos como el de 
Kyoto, que intentaba mitigar el calentamiento global, se ven restringidos por no vincular a todas las naciones en el mundo, como es el caso de países como China o India con economías en crecimiento -quienes no se encuentran obligados a formular ningún tipo de políticas que permitan reducir los gases de efecto invernadero- $y$ países que como Estados Unidos, que no están dispuestos firmar el protocolo porque limitaría su crecimiento económico. El protocolo de Kyoto sólo exige una reducción del $5 \%$ de emisiones de gases de efecto invernadero con respecto a lo emitido en 1990, y ni así es considerado por los países en mención. Adicionalmente, promueve los mecanismos de desarrollo limpio (MDL), los cuales consisten en promover que países en vía de desarrollo establezcan tecnologías más limpias que permitan la reducción de dióxido de carbono $\left(\mathrm{CO}_{2}\right)$. Estas reducciones luego son comercializadas como bonos de $\mathrm{CO}_{2}$ a países desarrollos. Este tipo de soluciones evidencia cómo se abordan los problemas ambientales desde una lógica económica que se materializan en soluciones técnicas dejando de lado la causa esencial del problema, que surge de la manera como nos relacionamos con la naturaleza ${ }^{13}$. Al igual que el protocolo de Kyoto, el protocolo de Cartagena sobre la seguridad de la biotecnología, realizado en el 2000 , convenio sobre la biodiversidad biológica, que pretende preservar la diversidad y la salud humana basándose en el principio de la precaución, no ha podido entrar en vigor, debido a la no participación de todas las naciones y de las multinacionales en el mismo.

\section{ELEMENTOS CRÍTICOS}

En conclusión, los principales problemas ambientales son producto de la manera como cubrimos las necesidades básicas de sobrevivencia y de las necesidades creadas por un medio social y económico que define gustos y determina elecciones de consumo. En general (excluyendo algunas naciones) las necesidades son enmarcadas dentro de un modelo de desarrollo cuyos fundamentos se encuentran en la capacidad de acumulación de capital, dejando en manos del mercado el bienestar humano. Este modelo de desarrollo adicionalmente propone sacrificar el presente para tener un futuro mejor ${ }^{14}$ (justificando la pobreza). A nivel ambiental es un absurdo pensar que la explotación irracional de los recursos a corto plazo traerá beneficios a largo plazo, pues el degradar la naturaleza tendrá como consecuencia un desequilibrio ambiental que no sólo nos vulnera como especie sino que atenta contra la vida en el planeta.

El actual modelo de desarrollo que nace dentro del marco de la modernización genera un modelo lineal, unidireccional, homogéneo y hegemónico, el cual utiliza la naturaleza para someterla a la economía de mercado, conduciéndola a la muerte. Las ideas de innovación y progreso centradas en la acumulación de capital reproducen el modelo capitalista que beneficia a un sector de la población, sacrificando a los otros y al ambiente. Lo anterior nos motiva a transformar la visión de la Tierra como un capital que provee de manera gratuita (en términos económicos) una serie de servicios a la especie humana, y comprender que hacemos parte del ciclo de la vida, donde la Tierra es el lugar de generación de la vida, la cual nos nutre de sus riquezas para mantener la extraordinaria diversidad de la cual goza nuestro planeta, donde los seres humanos somos un eslabón más y que hacemos parte del ciclo de vital. Este planteamiento nos exige un cambio en los patrones de consumo, que se traduce en un cambio en el estilo de vida. De esta manera es indispensable reevaluar las construcciones políticas económicas y el modelo capitalista, en donde el mercado anárquico es quien determina la pauta dentro del modelo de desarrollo, lo que oculta los riesgos ambientales a los que nos encontramos expuestos. 
Mientras los tratados ambientales no contemplen la crisis generada por el mercado, serán simples documentos de consulta para la elaboración de normas aplicadas a los efectos del deterioro ambiental, que en muchos de los casos ni aplican por la ausencia de voluntad de los estados que más contaminan, razón por la cual no definen en lo más mínimo la continuidad de la vida en el planeta.

\section{BIBLIOGRAFÍA}

- Declaración de la Conferencia de las Naciones Unidas sobre el Medio Ambiente, Reunidas en Estocolmo del 5 al 16 de Junio de 1972.

- Foro y Dialogo de Saberes Cambio Climático y Hoja de AYO-KOKHA, $3^{\text {er }}$ Festival Latinoamericano de Cine sobre Medio Ambiente, Septiembre 2008.

- Hissong, Robin. Las Teorías y las prácticas de desarrollo desde la perspectiva de la modernidad, Cuadernos Ocasionales 10, Centro Interdisciplinario de Estudios Regionales CIDER, Bogotá, 1996.

- Horkheimer, M.; Adorno, W. Theodor. Dialéctica de la ilustración Fragmentos filosóficos, introducción y traducción: Juan José, Sánchez. 3a. edición, Trotta, 1998.

- Informe Brundtland de las Naciones Unidas, Nuestro Futuro Común, 1987.

- Meadows, Dennis L. Los límites del crecimiento, trad. Cast. Loaeza Ma. Fondo de cultura económica, Bogotá, 1976.

- Método y conclusiones de la Evaluación de los Ecosistemas del Milenio. Diapositiva No. 5. CBD, Río Branco. Abril 2008.

- Roegen, Georgescu. La ley de la Entropía y los Procesos Económicos, Fundación Visor, Argentina, 1996.

- Sen, Amartya, "Las Teorías del Desarrollo Principios de Siglo XX", en: Cuadernos de Economía v. XII, No. 29, Universidad Nacional de Colombia, Bogotá, 1998

- Vandana, Shiva. Cosecha robada, El secuestro del suministro mundial de alimentos, Paidos, España, 2003.

- Naciones Unidas, Protocolo de Kyoto, 1998.

- Programa de las Naciones Unidas para el Medio Ambiente, Protocolo de Montreal, 2000.

\section{NOTAS}

1. El reconocimiento de la crisis ambiental no es desconocida ni siquiera para la conciencia "ingenua". El merito no está en describir el fenómeno y medirlo, sino en explicarlo y develar su devenir, lo que permite a la conciencia formada en la ciencia crítica la transformación racional de lo real, reconciliando así el pensamiento con la realidad, permitiendo al hombre encontrar su sentido en la tierra, su libertad.

2. Para el momento en el que es publicado el texto los límites del crecimiento era totalmente novedoso la modelación de sistemas en una computadora, cuestión que hoy es ampliamente utilizada en los más diversos análisis de sistemas.

3. En los límites de crecimiento se pone en consideración las siguientes variables: población, producción de alimentos, contaminación ambiental, industrialización y agotamiento de recursos no renovables. Meadows, Dennis L. Los límites del crecimiento, trad. Cast. Loaeza Ma. Fondo de cultura económica, Bogotá, 1976, p. 42. 
4. Op. cit., p. 115.

5. Las leyes de la termodinámica hacen referencia a las normas de comportamiento de la energía, las cuales permiten entender el permanente cambio del todo en el universo. La segunda ley, la de la entropía, indica que la energía libre (ordenada, disponible), pasa a energía dependiente o disipada, la cual ya no puede ser utilizada. La energía disponible se va incrementando de manera permanente dentro de todo sistema, pues si no fuera así una misma fuente de energía podría ser utilizada una y otra vez y no existiría la escasez. A pesar de ello, los seres vivos se han ordenado de tal forma que pueden evitar la degradación entrópica de su propia estructura. Piénsese por ejemplo en una ameba que ha resistido la labor destructiva durante casi dos mil millones de años. Esto sucede aunque cualquier sistema vivo da lugar al aumento de la entropía dentro de un sistema más rápidamente que otra situación. Al respecto ver Roegen, Georgescu. La ley de la Entropía y los Procesos Económicos, Fundación Visor, Argentina, 1996, p. 50 y ss. Sin embargo, dentro de los ecosistemas por medio de la cadena trófica, la naturaleza evita el aceleramiento del proceso entrópico, dado que todo residuo es fuente de alimento para miles de microorganismos que potencian la vida del suelo y con la fuente de energía primaria del planeta, el sol, permite que la vida se reproduzca de manera permanente en la tierra.

6. El modelo sobre el que el Club de Roma desarrolla su propuesta se basa en los niveles de consumo de la sociedad norteamericana -la más alta del planeta- y en los modos de producción agrícola de dicha sociedad, los cuales son ineficientes como lo demuestra el estudio de la Asociación Internacional de Fitocultivadores, sobre la comparación de policultivos tradicionales y los monocultivos industriales, en donde se muestra que en un sistema de policultivo pueden producirse 100 unidades de comida a partir de 5 unidades de input, mientras que en un sistema industrial requiere 300 unidades de input para producir esas mismas 100 unidades. Con las 295 unidades de recursos desperdiciados podrían haber producido 5.900 unidades de comida. ASSINSEL (Asociación Internacional de Fitocultivadores), "Feeding the 8 Billion and Preserving the Planet". En Vandana, Shiva. Cosecha robada, El secuestro del suministro mundial de alimentos, Paidos, España, 2003, p. 25.

7. Declaración de la Conferencia de las Naciones Unidas sobre el Medio Ambiente, Reunidas en Estocolmo del 5 al 16 de Junio de 1972, p. 1.

8. Bacon, In Praise of Knowledge, Miscellaneous Tracts upon Human Philosophy, en The Works of Francis Bacon, a cargo de Basil Montagu, en el concepto del Iluminismo Horkheimer, M.; Adorno, W. Theodor.Dialéctica de la ilustración Fragmentos filosóficos, introducción y traducción: Juan José, Sánchez. 3a. edición, Trotta, 1998, p. 6.

9. Declaración de la Conferencia de las Naciones Unidas sobre el Medio Ambiente, Reunidas en Estocolmo del 5 al 16 de Junio de 1972, p.1y 2.

10. Informe Brundtland de las Naciones Unidas, Nuestro Futuro Común, 1987.

11. Hissong, Robin (1996) "Las Teorías y las prácticas de desarrollo desde la perspectiva de la modernidad", Cuadernos Ocasionales 10, Centro Interdisciplinario de Estudios Regionales CIDER, Santa fe de Bogotá, p. 48.

12. Según la Evaluación de los Ecosistemas del Milenio del 2008 "durante los últimos 50 años, los humanos hemos cambiado la estructura y el funcionamiento de 
los ecosistemas más rápida y extensivamente que en cualquier período comparable de la historia, principalmente para obtener alimentos, agua, madera, energía y fibras. La mayor parte de los servicios de los ecosistemas están siendo degradados o usados de manera no sostenible". Lo anterior coloca en evidencia que el cubrir nuestras necesidades ha conllevado a una insostenibildad ambiental, lo cual nos exige un cambio en nuestros patrones de consumo. En Método y conclusiones de la Evaluación de los Ecosistemas del Milenio. Diapositiva No. 5. CBD, Río Branco, Abril 2008.

13. Comunidades indígenas han propuesto como MDL la reforestación evitada, solución que no se ha tenido en cuenta dado que eso implica dejar de explotar zonas naturales que traen altos beneficios económicos a quienes extraen materia de la misma. Foro y Dialogo de Saberes Cambio Climático y Hoja de AYO-KOKHA, $3^{\text {er }}$ Festival Latinoamericano de Cine sobre Medio Ambiente, Septiembre 2008.

14. Sen, Amartya, "Las Teorías del Desarrollo Principios de Siglo XXl", en: Cuadernos de Economía v. XII, No. 29, Universidad Nacional de Colombia, Bogotá, 1998, p. 79. 